\title{
Impact of oxygen therapy to ameliorate contrast-induced nephropathy in patients with acute coronary syndrome undergoing emergency angiography; a double-blinded clinical trial
}

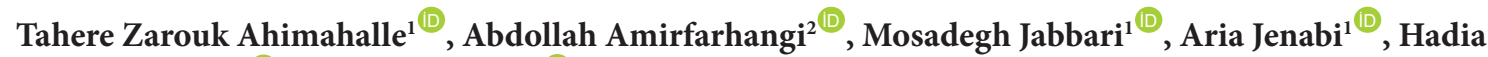 \\ Bagherzadegan $^{1(\mathbb{D}}$, Giti Noghabaei ${ }^{*}$ (D) \\ ${ }^{1}$ Internal Medicine Department, Iran University of Medical Sciences, Tehran, Iran \\ ${ }^{2}$ Department of Cardiology, Iran University of Medical Sciences, Tehran, Iran \\ ${ }^{3}$ Rasoul Akram Hospital, Iran University of Medical Sciences, Tehran, Iran
}

\section{A R T I C L E I N F O}

\section{Article Type:}

Original

\section{Article History:}

Received: 10 June 2019

Accepted: 15 August 2019

Published online: 13 September 2019

\section{Keywords:}

Oxygen therapy

Contrast nephropathy

Acute coronary syndrome

Emergent angiography

Chronic kidney disease

Hypertension

\begin{abstract}
A B S T R A C T
Introduction: Contrast-induced nephropathy (CIN) is one of the major causes of acute kidney injury.

Objectives: Regarding an increase in mortality and morbidity in patients with CIN, this study aimed to evaluate the effect of oxygen therapy in prevention of the CIN in individuals with acute coronary syndrome undergoing emergent angiography.

Patients and Methods: This study was a double-blinded clinical trial with control group (parallel design), randomized, and with a sample size of 204 individuals conducted on male or female patients over 35 years old and suspected of coronary artery disease undergoing emergent angiography refereed to Rasoul Akram hospital in 2018. Participants were divided into 2 groups (supplementary oxygen and oxygen-free groups). The first group received two to three liters of oxygen per minute from 10 minutes before the start of the procedure until the end of the procedure, and the second group inhaled the oxygen in the room air. Arterial blood gas (ABG) was taken prior to receiving oxygen and at the end of the procedure. Serum creatinine level was tested for all individuals before and 48 hours after the procedure.

Results: The mean age in intervention and control groups was $61.66 \pm 14.64$ years and $60.49 \pm 11.59$ years, respectively $(P=0.54)$. Mean glomerular filtration rate $(\mathrm{GFR})$ and serum creatinine before and after angiography was not significantly different $(P>0.05)$. There was a significant difference between the two genders regarding the development of CIN $(P=0.002)$, which was higher in women from that of men. Female gender was a strong risk factor and approximately increased four times the risk of CIN $(\mathrm{OR}=4.1 ;(P=0.001)$. History of chronic kidney disease $(\mathrm{CKD})$ and hypertension $(\mathrm{HTN})$ also produced such a situation $(\mathrm{OR}=22.37 ; P=0.007)$.

Conclusion: According to the results, oxygen therapy has no effect on the occurrence of CIN. It is also found that female gender, history of CKD and hypertension are risk factors for CIN.

Trial Registration: Registration of trial protocol has been approved in Iranian registry of clinical trials (\#IRCT20190317043082N1; https://en.irct.ir/trial/38486, Ethical code \#IR.IUMS.FMD. REC.1397.238).
\end{abstract}

Implication for health policy/practice/research/medical education:

Recent studies have suggested that the addition of supplemental oxygen to hydration leads to a significant reduction in CIN development. Reducing the risk of CIN with oxygen supplementation has been very much taken into consideration because of its safe nature for the patients and its availability. This study showed that oxygen therapy has no effect on the occurrence of CIN before and during emergency angiography. Based on small-sized samples and a set of studies in one center, it is required to study in a larger population and multiple centers for determination of using supplementary oxygen in the prevention of CIN.

Please cite this paper as: Ahimahalle T, Amirfarhangi A, Jabbari M, Jenabi A, Bagherzadegan H, Noghabaei G. Impact of oxygen therapy to ameliorate contrast-induced nephropathy in patients with acute coronary syndrome undergoing emergency angiography; a double-blinded clinical trial. J Renal Inj Prev. 2019;8(4):283-288. DOI: 10.15171/jrip.2019.52. 


\section{Introduction}

Contrast-induced nephropathy (CIN) is one of the major causes of acute kidney injury (1), which is reversible in most of the patients and $0.06 \%$ of the patients require renal replacement therapy (2). However, it should be taken into account that the mortality rates of the patients who have CIN are remarkably high (3). The definition of CIN is based on the special criteria including creatinine levels greater than or equal to $0.5 \mathrm{mg} / \mathrm{dL}$ or increased creatinine by more than $25 \%$ of a baseline level and having a time correlation with the injection of the contrast agent (48-72 hours after the injection of the contrast agent) in the absence of an alternative etiology (4). The first 24 hours after the injection of the contrast agent plays a very important role in the progression of CIN. In some studies, $80 \%$ of patients with CIN experienced an increase in creatinine within 24 hours and nearly all of them progressed to a serious renal failure $(5,6)$. The overall incidence of $\mathrm{CIN}$ in the general population is $0.6 \%$ to $2.3 \%$ (7). The incidence of CIN in people over 70 years old is $9.2 \%(8)$. In a prospective study on patients who received medical and surgical services, it showed that approximately $11 \%$ of kidney failure cases can be attributed to CIN following angiography, angioplasty and CT scan (9). The pathophysiology of CIN is poorly understood (10). Renal hypoxia and subsequently reactive oxygen species (ROS), vasoconstriction and inflammation have all been reported in various studies as factors that play an important role in the pathogenesis of CIN (11). Recent studies show that after exposure to the contrast, blood circulation is reduced by up to $40 \%$ to outer medulla, and then oxygenation is reduced up to $60 \%$ resulting in ischemic changes as a result of histopathology findings in CIN $(12,13)$. The participation of inflammatory cells and cytokines is also mentioned in the mechanism of CIN (14).

Risk factors for CIN are classified into two changeable and unchangeable factors. Unchangeable factors include advanced age, diabetes mellitus, pre-existing renal failure, advanced CHF, low LVEF (left ventricular ejection fraction), acute myocardial infarction, cardiogenic shock and renal transplant. Changeable factors include volume of contrast medium (CM), hypotension, anemia and blood loss, dehydration, low serum albumin levels, ACE inhibitors, diuretics, non-steroidal anti-inflammatory drugs, nephrotoxic antibiotics and IABP (intra-aortic balloon pump) (4). Regarding an increase in mortality and morbidity in patients with CIN, in a large retrospective study of more than 16,000 patients, 183 cases of CIN were detected. Although in this study, the incidence of CIN was approximately $2 \%$, the risk of mortality was $34 \%$, whereas in the control group that did not have CIN, this risk was $7 \%$. Additionally, with the adjustment of other associated confounding factors, it was found that CIN increases the risk of mortality by 5.5 times (15). Since CIN begins immediately after the exposure to the contrast agent, it is assumed that adequate oxygenation before exposure to the contrast accompanied with hydration by isotonic saline can prevent CIN more effectively. Recent studies have also suggested that the addition of supplemental oxygen to hydration leads to a significant reduction in CIN development from that of hydration alone $(16,17)$.

\section{Objectives}

The idea of reducing the risk of CIN with oxygen supplementation has been very much taken into consideration because of its safe nature for the patient, its ease of administration, and its availability. We evaluated the incidence of CIN in patients who had received supplemental oxygen in comparison with patients who had undergone only hydration.

\section{Patients and Methods \\ Protocol}

This study was a double-blinded clinical trial with control group (parallel design), randomized, with a sample size of 204 individuals conducted on male or female subjects over 35 years old and supposed having coronary artery disease undergoing emergent angiography refereed to Rasoul Akram hospital in 2018 (Figure 1). Exclusion criteria were arterial oxygen saturation less than $90 \%$, having end-stage kidney failure, patients undergoing dialysis and candidate for emergency coronary artery bypass surgery. The patients were divided into two groups.

The random number table and block randomization method were used using http://www.randomizer.org. In this method, eligible patients are divided into blocks of four patients. Random numbers were created using a computer. Based on the determined numerical range to enter patients in each group, half of the patients assigned to supplementary oxygen and half of them in another group. All participants were first visited and their creatinine level and arterial blood gas (ABG) were measured in emergency department of Rasoul Akram hospital. The individuals were randomly divided into two groups; supplementary oxygen receiving group and the oxygen free group. The first group received two to three liters of oxygen per minute from 10 minutes before the start of the procedure until the end of the procedure, and the second group inhaled the oxygen in the room air. ABGs were obtained prior to receipt of oxygen and at the end of the procedure, and all ABGs were sent to the laboratory of that center. Control group did not receive supplementary oxygen. This group inhaled the oxygen in the room air. All participants were given a same contrast. The dose of the contrast agent was at least $100 \mathrm{~mL}$ and maximum $200 \mathrm{~mL}$. Serum creatinine level was assessed for all individuals before and 48 hours after the procedure. Glomerular filtration rate (GFR) was measured using MDRD (Modification of Diet in Renal Disease) $\left(\mathrm{mL} / \mathrm{min} / 1.73 \mathrm{~m}^{2}\right)$. 


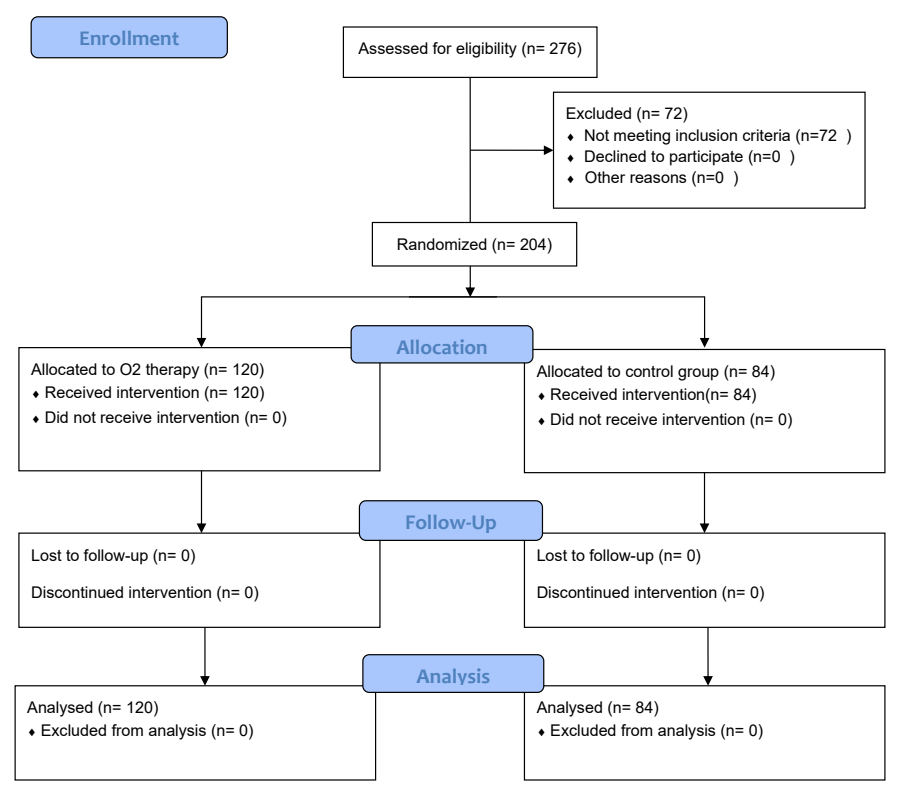

Figure 1. Diagram of the study.

\section{Ethical issues}

This investigation is in accordance with the Helsinki Declaration and approved by Ethical Committee of Iran University of Medical Sciences \#IR.IUMS.FMD. REC.1397.238) and by Iranian randomized clinical trial (\#IRCT20190317043082N1; https://en.irct.ir/trial/38486). The registration number of thesis is 2947 . This study was conducted as the internal medicine residency thesis of Giti Noghabaei at this university.

\section{Statistical analysis}

The results for quantitative variables are expressed as mean and standard deviations (mean \pm SD) and for the qualitative variables, expressed as percentages. Comparison between quantitative variables was performed by independent t-test and also for qualitative variables, using chi-square test. Correlation between quantitative variables was investigated using Pearson's correlation test. For determining the difference in the frequency of nephropathy in the two groups and in the presence of basic features of patients as confounding factors of the study, multivariate logistic regression analysis was used and the results were expressed as odds ratio (95\% confidence interval). SPSS 21 was used for statistical analysis. A $P$ value less than 0.05 was considered significant.

\section{Results}

In this study, 204 patients were evaluated, 120 patients were treated with supplementary oxygen and 84 patients without supplemental oxygen. There was no significant difference between the two groups regarding the mean age, mean ejection fraction (EF), frequency of diabetes mellitus and the frequency of chronic kidney disease $(\mathrm{CKD})$, diuretics consumption and ACE inhibitors $(P>$
0.05). In the group treated with supplementary oxygen, $75 \%$ were male and $25 \%$ were female, and in the control group, $86.9 \%$ were male and $23.1 \%$ were female. The mean age in the intervention and control groups was $61.66 \pm$ 14.64 years and $60.49 \pm 11.59$ years, respectively, which did not show a significant difference $(P=0.54)$. Accordingly, mean EF (ejection fraction) was $36.96 \pm 12.87 \%$ and $39.56 \pm 12.38 \%$, respectively, which did not show a significant difference $(P=0.17)$. Mean creatinine before angiography was $1.63 \pm 1.11 \mathrm{mg} / \mathrm{dL}$ and $1.52 \pm 0.71 \mathrm{mg} / \mathrm{dL}$, respectively, which did not show a significant difference $(P=0.41)$. The mean creatinine after angiography was $1.58 \pm 0.83$ and $1.51 \pm 0.92$ in the intervention group, which did not show a significant difference between the two groups $(P=0.53)$ (Figure 2, Table 1). Mean GFR before angiography was $50.11 \pm 18.65 \mathrm{~mL} / \mathrm{min} / 1.73 \mathrm{~m}^{2}$ and $52.58 \pm 19.30 \mathrm{~mL} / \mathrm{min} / 1.73 \mathrm{~m}^{2}$, respectively, which did not show a significant difference $(P=0.36)$ as well as the

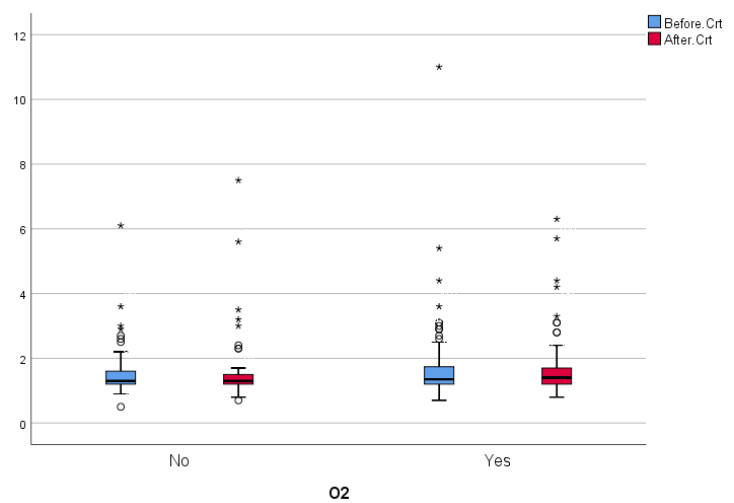

Figure 2. Serum creatinine level before and after angiography in patients with and without supplementary oxygen therapy. 


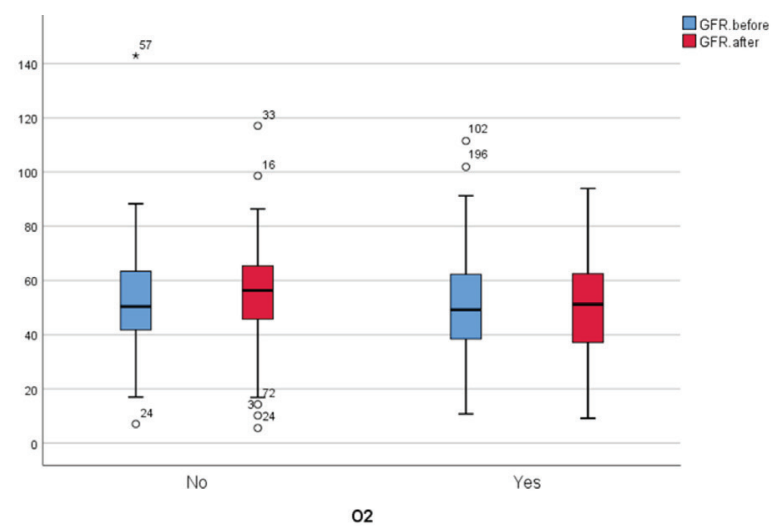

Figure 3. GFR before and after angiography in patients with and without supplementary oxygen therapy.

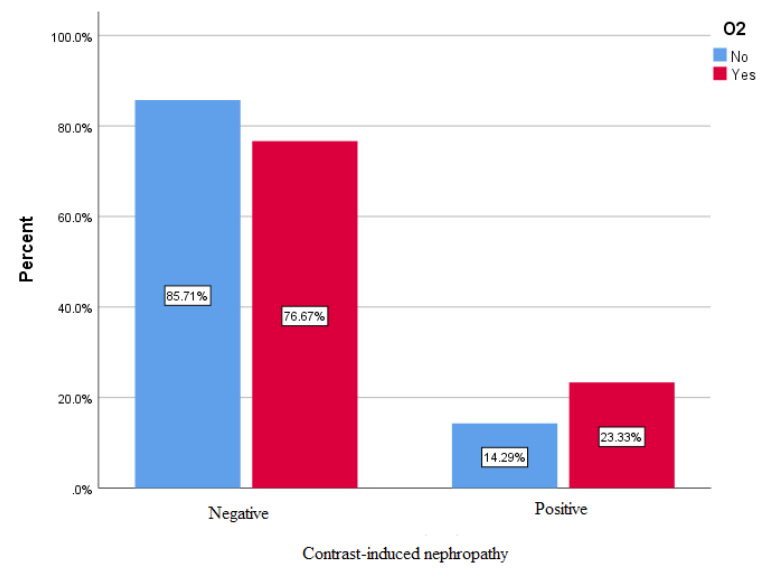

Figure 4. Prevalence of contrast-induced nephrophathy in patients with and without supplementary oxygen therapy.

mean GFR after the intervention in the both groups were $49.96 \pm 18.85 \mathrm{~mL} / \mathrm{min} / 1.73 \mathrm{~m}^{2}$ and $54.24 \pm 17.57 \mathrm{~mL} / \mathrm{mi}$ Figure $\mathrm{n} / 1.73 \mathrm{~m}^{2}$, respectively, which was not statistically significant $(P=0.10$; Figure 3 , Table 1$)$, but having hypertension showed a significant difference between the two groups $(P>0.05)$. The mean GFR changes in the two groups were $17.97 \pm 0.17 \mathrm{~mL} / \mathrm{min} / 1.73 \mathrm{~m}^{2}$ and $17.4 \pm 1.65$ $\mathrm{mL} / \mathrm{min} / 1.73 \mathrm{~m}^{2}$, respectively, which did not show a statistically significant difference $(P=0.46)$. Based on the definition of CIN, its prevalence in the intervention group was $23.3 \%$ (28 cases) and in the control group $14.3 \%$ (12 patients). There was no significant difference between the two groups $(P=0.109$; Figure 4$)$. The prevalence of contrast induced nephropathy in men was $15.3 \%(n=25)$ and in women was $36.6 \%(n=15)$. There was a significant difference between the two genders $(P=0.002)$, which was higher in women from that of men.

The pevalence of CIN in patients with $(n=7)$ and without $(\mathrm{n}=197)$ CKD was $71.4 \%$ and $17.2 \%$ respectively, which showed a significant difference between two groups. $(P=0.001$; Figure 5).

To prevent estimation bias, variables with significance level less than 0.1 were entered in the logistic model. Link function was logit with significant fitting $(P<0.05)$ and large goodness of fit $(P>0.05)$. In binary logistic regression analysis, gender was presented as a risk factor in the model, that is, by shifting gender from male to female, provided that the other variables remain constant in the model. Thus, approximately four times the risk of CIN increases because the odds ratio $(\mathrm{OR}=4.1)$ is significant $(P=0.001)$. History of $\mathrm{CKD}$ and hypertension also produced such a situation $(\mathrm{OR}=22.37, P=0.007$; Table 2$)$.

\section{Discussion}

This study aimed to evaluate the effect of oxygen therapy before and during emergent angiography to reduce the incidence of CIN in patients under coronary angiography. Performing diagnostic and therapeutic procedures is one of the most important risk factors for the development of nephropathy caused by contrast agents. In this interventional study, the intervention group received 2-3 liters of oxygen per minute from 10 minutes before and during the angiography, and the serum creatinine level was evaluated 48 hours after the angiography. Our results showed that supplementary oxygen does not reduce the incidence of CIN. Meanwhile, female gender, history of

Table 1. The mean GFR and creatinine before and after intervention in the two groups

\begin{tabular}{|c|c|c|c|c|}
\hline \multirow{2}{*}{ Group } & \multicolumn{2}{|c|}{ GFR (mean \pm SD) } & \multicolumn{2}{|c|}{ Creatinine (mean \pm SD) } \\
\hline & Before intervention & After intervention & Before intervention & After intervention \\
\hline Intervention & $50.11 \pm 18.65$ & $49.96 \pm 18.85$ & $1.63 \pm 1.11$ & $1.58 \pm 0.83$ \\
\hline Control & $52.58 \pm 19.30$ & $54.24 \pm 17.57$ & $1.52 \pm 0.71$ & $1.51 \pm 0.92$ \\
\hline$P$ value & 0.36 & 0.1 & 0.53 & 0.41 \\
\hline
\end{tabular}

Table 2. Predictive variable for development of $\mathrm{CIN}$ (as a dichotomous variable) using logistic regression

\begin{tabular}{lccccc}
\hline Variable & Odds ratio & Standard error & $P$ value & Lower limit \\
\hline History of CKD & 22.3 & 5.37 & 0.007 & 20.818 \\
Sex & 4.1 & 2.241 & 0.001 & 3.078 & 4.385 \\
\hline
\end{tabular}




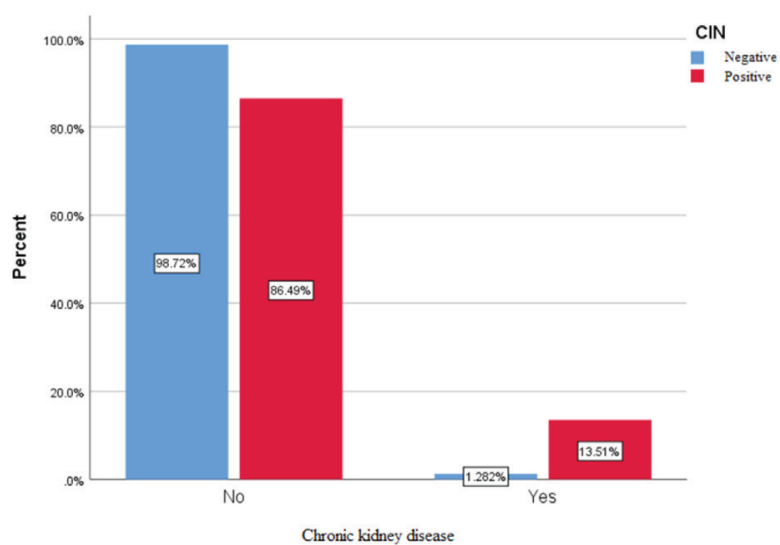

Figure 5. Prevalence of contrast-induced nephrophathy in patients with and without chronic kidney disease.

CKD and HTN were significantly associated with the development of CIN.

These findings were inconsistent with previous studies. In the study of Minoo et al, the prevalence of CIN in individuals undergoing oxygen therapy were significantly lower than control group (18.6\% versus $41.5 \%$ ) (17), while in the study of Sekiguchi et al, the results were the same with the study by Minoo et al (0.6\% versus $5.1 \%)$ (16). The type of procedure in our study was emergency, while the two previous studies have been performed electively and the hemodynamic status of the patients was stable, in such a way that the risk of CIN is less in elective patients from those of in emergency patients.

Observed contrasts can be based on the confounding factors in this study including the history of angioplasty and chronic heart failure, anemia, dehydration, precision of the administration of contrast agents and their viscosity, the administration of antibiotics and nephrotoxic drugs such as NSAIDs. Due to a defect in patients' records and the lack of accessibility to detailed information, the patients with the mentioned confounding were excluded from the study. Furthermore, in the study of Minoo et al, the prevalence of nephropathy was significantly higher in males undergoing angiography (odds ratio equal to 11.3), but in our study, the female gender was significantly strong predictor for developing CIN (odds ratio equal to 4.1).

Indeed, our study approved the effect of gender on the development of CIN (17). This study was one of the few studies conducted on the effect of oxygen therapy on nephropathy in patients undergoing angiography with more studied variables such as age, gender, EF, GFR, chronic renal disease, heart disease, diabetes, hypertension and ACE inhibitors and also diuretics, which was one of the strengths of this research. In a study by Rubinstein et al, renal failure was induced to a few numbers of laboratory mice and then treated with $100 \%$ oxygen. The study showed that renal blood flow in mice treated with oxygen was much higher from that in untreated mice.
Likewise, GFR decreased by $68 \%$ in mice treated with oxygen and $94 \%$ in oxygen-free mice (18). These results were not consistent with the results of our study.

\section{Conclusion}

This study showed that oxygen therapy has no effect on the occurrence of contrast-induced nephropathy before and during emergency angiography. It is also found that female gender and history of CKD and hypertension are risk factors for contrast-induced nephropathy.

\section{Limitations of the study}

Limitations of this study can be small sample size, the effect of other factors such as metabolic syndromes, anemia, hypoalbuminemia, dehydration, precision of the use of contrast agents and their viscosity, and the use of antibiotics and other nephrotoxic drugs such as Nonsteroidal antiinflammatory drugs, and kidney transplant. Additionally, for the diagnosis of nephropathy, only serum creatinine changes have been established, and other kidney injury biomarkers such as urine volume, proteinuria or cystatin C were not evaluated. Other limitation of this study was that the information is collected from a single center.

\section{Authors' contribution}

TZ designed the study, performed analysis and interpretation of data. AJ, MJ and $\mathrm{HB}$ edited the draft. AA performed emergent coronary angiography. GN gathered the data and wrote the primary manuscript. All authors read, revised, and approved the final manuscript.

\section{Conflicts of interest}

None.

\section{Ethical considerations}

Ethical issues (including plagiarism, data fabrication, double publication) have been completely observed by the authors.

\section{Funding/Support}

This research did not receive any specific grant from agencies in the public, commercial, or not for profit sectors. The registration number of thesis is 2947.

\section{References}

1. Chen YH, Fu YC, Wu MJ. Does Resveratrol Play a Role in Decreasing the Inflammation Associated with Contrast Induced Nephropathy in Rat Model? J Clin Med. 2019;8:147. doi: $10.3390 / \mathrm{jcm} 8020147$.

2. Kooiman J, Pasha SM, Zondag W, Sijpkens YW, van der Molen AJ, Huisman MV, et al. Meta-analysis: serum creatinine changes following contrast enhanced CT imaging. Eur J Radiol. 2012;81:2554-61. doi: 10.1016/j. ejrad.2011.11.020.

3. Weisbord SD, Palevsky PM. Radiocontrast-induced acute renal failure. J Intensive Care Med. 2005;20:63-75. doi: 


\section{$10.1177 / 0885066604273503$.}

4. Mehran R, Nikolsky E. Contrast-induced nephropathy: definition, epidemiology, and patients at risk. Kidney Int Suppl. 2006;69:11-5. doi: 10.1038/sj.ki.5000368.

5. Stevens MA, McCullough PA, Tobin KJ, Speck JP, Westveer DC, Guido-Allen DA, et al. A prospective randomized trial of prevention measures in patients at high risk for contrast nephropathy: results of the P.R.I.N.C.E. Study. Prevention of Radiocontrast Induced Nephropathy Clinical Evaluation. J Am Coll Cardiol. 1999;33:403-11. doi: 10.1016/s07351097(98)00574-9.

6. Guttterez NV, Diaz A, Timmis GC, O’neill WW, Stevens MA, Sandberg KR, et al. Determinants of serum creatinine trajectory in acute contrast nephropathy. J Interv Cardiol. 2002;15:349-54.

7. Lasser EC, Lyon SG, Berry CC. Reports on contrast media reactions: analysis of data from reports to the US Food and Drug Administration. Radiology. 1997;203:605-10. doi: 10.1148/radiology.203.3.9169676.

8. Levey AS, Coresh J, Balk E, Kausz AT, Levin A, Steffes MW,et al. National Kidney Foundation practice guidelines for chronic kidney disease: evaluation, classification, and stratification. Ann Intern Med. 2003;139:137-47. doi: 10.7326/0003-4819-139-2-200307150-00013.

9. Nash K, Hafeez A, Hou S. Hospital-acquired renal insufficiency. Am J Kidney Dis. 2002;39:930-6. doi: 10.1053/ajkd.2002.32766.

10. Persson PB, Hansell P, Liss P. Pathophysiology of contrast medium-induced nephropathy. Kidney Int. 2005;68:14-22. doi: 10.1111/j.1523-1755.2005.00377.x.

11. Gao Z, Han Y, Hu Y, Wu X, Wang Y, Zhang X, et al. Targeting $\mathrm{HO}-1$ by epigallocatechin-3-gallate reduces contrast-induced renal injury via anti-oxidative stress and anti-inflammation pathways. PLoS One. 2016;11:e0149032. doi: $\quad$ 10.1371/journal.pone.0149032.

12. Liss P, Nygren A, Olsson U, Ulfendahl HR, Erikson U. Effects of contrast media and mannitol on renal medullary blood flow and red cell aggregation in the rat kidney. Kidney Int. 1996;49:1268-75.

13. Nygren A. Contrast media and regional renal blood flow. A study of the effects of ionic and non-ionic monomeric and dimeric contrast media in the rat. Acta Radiol Suppl. 1992;378:123-35.

14. Andreucci M, Lucisano G, Faga T, Bertucci B, Tamburrini $\mathrm{O}$, Pisani A, et al. Differential activation of signaling pathways involved in cell death, survival and inflammation by radiocontrast media in human renal proximal tubular cells. Toxicol Sci. 2011;119:408-16. doi: 10.1093/toxsci/ kfq332.

15. Levy EM, Viscoli CM, Horwitz RI. The effect of acute renal failure on mortality: a cohort analysis. JAMA. 1996;275:1489-94.

16. Sekiguchi H, Ajiro Y, Uchida Y, Ishida I, Otsuki H, Hattori $\mathrm{H}$, et al. Oxygen pre-conditioning prevents contrastinduced nephropathy (OPtion CIN Study). J Am Coll Cardiol. 2013 ;62:162-163. doi: 10.1016/j.jacc.2013.04.012.

17. Bhandari P, Shah Z, Patel K, Patel R. Contrast-induced acute kidney injury following coronary angiography in patients with end-stage liver disease. J Community Hosp Intern Med Perspect. 2019;9(5):403-9. doi: 10.1080/20009666.2019.1661148.

18. Rubinstein I, Abassi Z, Milman F, Ovcharenko E, Coleman $\mathrm{R}$, Winaver J, et al. Hyperbaric oxygen treatment improves GFR in rats with ischaemia/reperfusion renal injury: a possible role for the antioxidant/oxidant balance in the ischaemic kidney. Nephrol Dial Transplant. 2009; 24:428436. doi: $10.1093 / \mathrm{ndt} / \mathrm{gfn} 511$.

Copyright () 2019 The Author(s); Published by Nickan Research Institute. This is an open-access article distributed under the terms of the Creative Commons Attribution License (http://creativecommons.org/licenses/by/4.0), which permits unrestricted use, distribution, and reproduction in any medium, provided the original work is properly cited. 